Over 225000 new cases of ovarian cancer are diagnosed each year. Symptoms are often vague, so most cases are detected when the disease is at an advanced stage. There is a need to find new drugs which will be able to treat ovarian cancer effectively. One of the most promising antineoplastic agents is trabectedin (Yondelis), derived from the marine tunicate Ecteinascidia turbinata, approved by the European Union in July 2007 for the treatment of soft-tissue sarcomas. This drug shows a mechanism of action based on the inhibition of the nucleotide excision repair system. Trabectedin shows anti-tumour activity in vitro and in vivo in ovarian, breast, prostate, renal, melanoma and non-small cell lung cancer cell lines. Trabectedin in combination with pegylated liposomal doxorubicin demonstrates synergistic antineoplastic activity.

Key words: trabectedin, chemotherapy, pegylated liposomal doxorubicin, ovarian cancer.

Contemp Oncol (Pozn) 2014; 18 (3): 149-152 DOI: $10.5114 /$ wo. 2014.43153

\section{Trabectedin as a single agent and in combination with pegylated liposomal doxorubicin - activity against ovarian cancer cells}

\author{
Agnieszka Marczak, Marta Denel
}

Department of Thermobiology, Faculty of Biology and Environmental Protection, University of Lodz, Poland

\section{Introduction}

Ovarian cancer is called "the silent killer" because of its not-so-obvious symptoms such as fatigue, weight change, abdominal distention and pain. The lack of efficient and early detection is the reason for the high mortality rate. This neoplasm is responsible for 125000 deaths annually [1-3]. The "gold standard" of ovarian cancer treatment consists of surgery followed by carboplatin alone, or a combination of chemotherapy with carboplatin and paclitaxel (PTX). Currently, first line therapy in early disease (stages I-Ila) is only surgery. In $25 \%$ of patients, this protocol does not cure, and the disease will recur. In advanced stages (IIa-IV), the current standard of care is cytoreduction followed by platinum-based chemotherapy. Unfortunately, this treatment does not work for most women. Because of frequent relapses, most patients die [4]. Furthermore, side effects are considered a very important problem connected with chemotherapy. The most common adverse effects include haematological disorders, nausea, vomiting, disruption of the bone cycle and pain. According to most patients, alopecia is one of the most distressing side effects. Chemotherapeutic agents often cause the loss of hair. Drugs commonly used in ovarian cancer treatment (paclitaxel, docetaxel) cause severe alopecia which markedly lowers the quality of patients' lives [5, 6].

There is a need to investigate and find new drugs which will selectively and effectively treat ovarian cancer, and also be able to overcome multidrug resistance (MDR) - the factor responsible for many cases of recurrent epithelial ovarian cancer (EOC).

\section{Trabectedin - structure and mechanism of action}

Trabectedin, also known as Yondelis or ET-743, is an anti-tumour drug, originally derived from the marine tunicate Ecteinascidia turbinata. Now it is obtained from the antibiotic cynosafracin B [7]. It is composed of three fused tetrahydroisoquinoline rings (Fig. 1). Two of the rings covalently bind the $\mathrm{N}_{2}$ amino group in the guanine residue in the DNA minor groove, in contrast to traditional alkylating drugs that bind guanine at the N7 or 06 position in the DNA major groove. Favoured triplets are TGG, CGG, AGC, and GGC. The CGA triplet is refractory. The adducts are stabilized by van der Waals interactions and hydrogen bonds between rings $A$ and $B$ and DNA $[7,8]$. The third unbound $C$ ring affects critical nuclear proteins, mainly transcription factors. Trabectedin induces DNA alkylation and DNA-protein crosslinks which cause formation of DNA strand breaks [7, 9, 10]. The mechanism of action is based on inhibition of DNA transcription. Trabectedin causes disruption of transcription by inhibition of the transcription-dependent nucleotide excision repair system (NER), followed by G2/M arrest and activation of the extrinsic and intrinsic apoptotic pathways, occurring through p53-independent pro- 


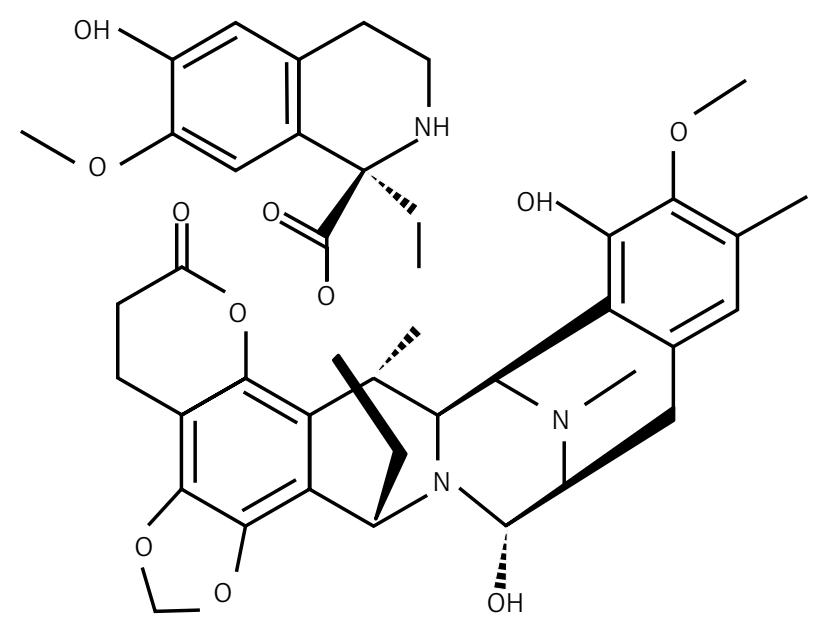

cess [7, 11, 12]. Data on HeLa, Chinese hamster and human leukaemia cells obtained by Soares et al. [13] indicated that DNA synthesis and cellular viability were reduced by $50 \%$ after $1 \mathrm{~h}$ exposure to, respectively, $30 \mathrm{nM}$ and $20 \mathrm{nM}$ trabectedin. It was associated with formation of difficult-to-repair drug-DNA adducts, converted later into DNA double-strand breaks (DSBs). The phosphorylated histone $\mathrm{H} 2 \mathrm{AX}(\gamma-\mathrm{H} 2 \mathrm{AX})$ was considered as a marker. Furthermore, trabectedin toxicity was 8-fold higher toward BRCA2 deficient cells compared with the parental cell lines. Unrepaired DSBs in BRCA2-deficient cells led to chromosomal aberrations [13]. Due to the promising outcomes of trabectedin, many of its natural derivatives were isolated from Ecteinascidia turbinata. Some of these analogues are presented in Table 1.

Fig. 1. Structure of trabectedin

Table 1. Natural derivatives of trabectedin isolated from Ecteinascidia turbinata (based on [20])

ET-759A

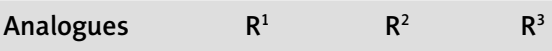

Analogues $R^{1}$ $\mathrm{R}^{2}$ $\mathrm{R}^{3}$ $\mathrm{R}^{4}$

ET-637

ET-594

ET-552

ET-652

$\begin{array}{cccc}\mathrm{Ac} & \mathrm{OH} & \mathrm{H} & \mathrm{NHAC} \\ \mathrm{Ac} & \mathrm{OH} & - & =\mathrm{O} \\ \mathrm{H} & \mathrm{OH} & - & =\mathrm{O} \\ \mathrm{Ac} & \mathrm{OH} & \mathrm{H} & \mathrm{NHCOCH}_{2} \mathrm{NH}_{2}\end{array}$

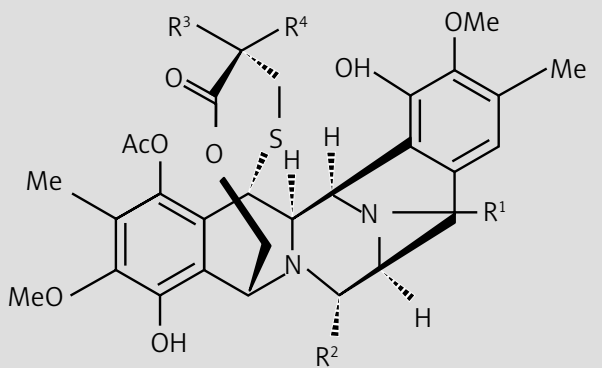

$\begin{array}{lcccc}\text { ET-583 } & \mathrm{H} & \mathrm{OH} & \mathrm{H} & \mathrm{NH}_{2} \\ \text { ET-597 } & \mathrm{CH}_{3} & \mathrm{OH} & \mathrm{H} & \mathrm{NH}_{2} \\ \text { ET-596 } & \mathrm{CH}_{3} & \mathrm{OH} & - & =\mathrm{O} \\ \text { ET-641 } & \mathrm{CH}_{3} & \mathrm{H} & \mathrm{H} & \mathrm{NHAC}\end{array}$




\section{Trabectedin as a single agent}

Trabectedin was approved in the European Union in July 2007 for the treatment of soft-tissue sarcomas (STS) in adults after failure of conventional therapy including anthracyclines and ifosfamide [7, 9, 11]. In a preclinical study, it demonstrated antineoplastic activity in vitro and in vivo in ovarian, breast, prostate, renal, melanoma and non-small cell lung cancer [11]. It is effective in ovarian carcinoma xenografts and ovarian cancer explants and can be combined with cisplatin [10]. Unfortunately, in vitro studies with mammalian cells transfected with MDR1 genes and an in vivo study in mice with P-gp overexpression showed that trabectedin is a substrate of P-glycoprotein (P-gp), the molecule responsible for multidrug resistance in human cancer cells [14].

Anti-tumour activity of trabectedin in ovarian cancer has been studied in phase I and phase II clinical trials [11]. The phase I trial study included women with platinum-sensitive and platinum-resistant ovarian cancer after therapy based on platinum and taxanes. Trabectedin caused 43\% response rates in patients with platinum-sensitive ovarian cancer, with a time to progression of 7 to 9 months. There were also observed two partial responses among women with platinum-resistant ovarian cancer. The time to progression was 5 months. The most common adverse effects were nausea and vomiting (78\%), neutropenia (41\%), asthenia (78\%) and thrombocytopenia (7.5\%) [15]. Phase II clinical trials included women with recurrent ovarian cancer after one or two platinum-based chemotherapy regimens. Trabectedin showed $29 \%$ and $6.3 \%$ response rates in women with platinum-sensitive and platinum-resistant ovarian cancer respectively, with a median progression time of between 5.1 and 2 months. The side effects were neutropenia (8\%), nausea, vomiting, and fatigue (5\%) [16].

Anti-tumour effectiveness of trabectedin in monotherapy was evaluated by Mabuchi et al. [11] in vitro using 3 lines of human ovarian clear cell carcinoma (CCC) and human ovarian serous adenocarcinomas (SAC) - 3 lines. It inhibited growth and proliferation of CCC and SAC. CCC showed higher sensitivity to trabectedin than SAC cell lines. The effect was maintained in cisplatin- and paclitaxel-resistant CCC cells. Then, the anti-tumour effect of trabectedin was evaluated in vivo using a xenograft model with mice inoculated with CCC cells. Trabectedin was generally well tolerated. After 4 weeks of treatment the tumour mass was reduced by greater than $70 \%$, compared with PBS-treated mice [11].

The drug is generally well tolerated. It is metabolized in the liver with a half-life of approximately 90 hours. Cytochrome P 450 CYP3A4 isoenzyme is involved in this process. The major tissue toxicities are connected with the bile duct (elevated plasma bilirubin, bile acids and aspartate transaminase (AST), alanine transaminase (ALT), $\gamma$-glutamyl transferase (GGT) and alkaline phosphatase activities). The most common side effects are fatigue, nausea, anorexia, vomiting, constipation, atelectasis, dyspnoea, neutropenia, haemorrhoids, and intestinal obstruction. There were no indications of renal toxicity in rats (or mice and dogs) $[7,14,17,18]$. There is also no cardiac toxicity and no ECG changes (considered as prolongation of QT/QTc interval) after treatment with trabectedin [17].

\section{Trabectedin in combination with pegylated doxorubicin}

Very interesting data were obtained for the combination of trabectedin with pegylated liposomal doxorubicin (PLD, Doxil). It is a unique form of anthracycline antibiotic - doxorubicin, packed in a liposome coated with polyethylene glycol. This modification prevents plasma protein adsorption to the liposome surface and showed that in contrast to non-pegylated liposomes, PLDs are able to remain in the circulation much longer. Due to the enhanced permeability and retention (EPR) effect, the liposomes delivered drugs more specifically to the cancer tissues and limited exposure of normal cells to the drugs. The use of PLD for ovarian cancer treatment gave promising results $[19,20]$.

Based on the results of a randomized phase III trial (ET-743-OVA-301) (comparing PLD alone with a combination of PLD and trabectedin), on 672 patients diagnosed with a recurrent ovarian cancer from 21 countries in 2009, the European Commission approved the combination with PLD: $30 \mathrm{mg} / \mathrm{m}^{2}$ (as a $3 \mathrm{~h}$ infusion), every 3 weeks, for the treatment of patients with a recurrent platinum-sensitive ovarian cancer, for whom a first line platinum-based chemotherapy had failed [14, 21, 22]. The study demonstrated that trabectedin in combination with PLD improves progression-free survival (PFS) and overall response rate (ORR) in comparison to Doxil alone as a second-line treatment of recurrent ovarian cancer. Among the 672 patients, 522 (77.7\%) deaths were observed (including 258 in the trabectedin and Doxil arm and 264 in the Doxil arm). The median overall survival (OS) for trabectedin plus Doxil and Doxil alone was 22.2 and 18.9 months respectively. This effect was observed in all age groups except $>65$. Surprisingly, the progression-free interval (PFI) favouring the PLD arm (PLD - PFI) was 13.3 months, whereas trabectedin + PLD - PFI was 10.6 months. Additionally, this combination was well tolerated, with manageable toxicity. Furthermore, a decrease of PLD-associated toxicity was observed, which supports the thesis that trabectedin in combination with PLD is a good solution for patients with recurrent ovarian cancers [21-23].

\section{Future combined therapies with trabectedin}

Angiogenesis - the creation of new blood and lymphatic vessels - is a crucial process for tumour development. Most types of tumours respond to a hypoxic environment by secreting a key pro-angiogenic growth factor called vascular endothelial growth factor (VEGF). These particles bind to its receptors (VEGFR-1, VEGFR-2, VEGFR-3) on the surface of cancer cells, leading to metastasis. Studies by Klasa-Mazurkiewicz et al. [24] showed that the overexpression of VEGFR-2 occurred among patients with early ovarian cancer stages (FIGO - I, Ila), which may indicate the important role of apoptosis during this phase, whereas an increased level of VEGFR-3 was detected in advanced stages of cancer, and correlated with a positive response to chemotherapy. A high level of VEGFR-3 is also connected with aggressiveness of ovarian cancer and indicates poor prognosis.

Angiogenesis is not the only mechanism of a tumour vasculature. Vasculogenic mimicry is a phenomenon con- 
nected with the formation of fluid-conducting channels, not lined with endothelium. This process occurs during tumour development and concerns undifferentiated cancers especially in the advanced stage of the disease. The presence of vasculogenic mimicry may indicate a poor prognosis. Additionally, some researchers claim that vasculogenic mimicry may protect the neoplasm against anticancer agents (as tumours demonstrating this phenomenon are often drug-resistant) [25].

Currently the most studied agent among anti-angiogenic drugs is bevacizumab - a humanized IgG1 monoclonal antibody which selectively inhibits VEGF activity. Two phase III clinical trials, GOG-218 and ICON7, showed a significant benefit in PFS when the standard (carboplatin-paclitaxel) chemotherapy was combined with bevacizumab. Based on these results, the European Medicines Agency (EMA) approved this combination of drugs for the front line treatment of advanced epithelial ovarian cancer [26]. Interestingly, studies by Takano et al. [27] indicated that bevacizumab in combination with trabectedin and oxaliplatin causes complete remission of recurrent ovarian clear cell carcinoma, with manageable general toxicity. It is thought that bevacizumab blocks vascular repair and survival, which enhances the activity of trabectedin and oxaliplatin.

The latest studies indicate that angiogenesis and vasculogenic mimicry are important in tumour development; therefore deeper understanding of the individual angiogenic patient's phenotype may be helpful in designing an appropriate and effective anticancer therapy.

\section{The authors declare no conflict of interest.}

\section{References}

1. Gentry-Maharaj A, Menon U. Screening for ovarian cancer in the general population. Best Pract Res Clin Obstet Gynaecol 2012; 26: 243-56.

2. Zhang B, Cai FF, Zhong XY. An overview of biomarkers for the ovarian cancer diagnosis. Eur J Obstet Gynecol Reprod Biol 2011; 158: 119-23.

3. Ng JS, Low JJ, Ilancheran A. Epithelial ovarian cancer. Best Pract Res Clin Obstet Gynaecol 2012; 26: 337-45

4. Pignata S, Cannella L, Leopardo D, Pisano C, Bruni GS, Facchini G. Chemotherapy in epithelial ovarian cancer. Cancer Lett 2011; 303: 73-83.

5. Dąbrowski T. Hair loss as a consequence of cancer chemotherapy - physical methods of prevention. A review of the literature. Contemp Oncol (Pozn) 2011; 15: 95-101.

6. Leśniewski-Kmak K, Radecka B, Litwiniuk M. Bone tissue as the site of action of oncological drugs. Contemp Oncol (Pozn) 2011; 15: 147-54.

7. Christinat A, Leyvraz S. Role of trabectedin in the treatment of soft tissue sarcoma. Onco Targets Ther 2009; 2: 105-13.

8. D'Incalci M, Galmarini CM. A review of trabectedin (ET-743): a unique mechanism of action. Mol Cancer Ther 2010; 9: 2157-63.

9. Koseła H, Wiater K, Świtaj T. Trabectedin in pateints with pre-treated advanced soft tissue sarcoma: a retrospective single center analysis. Contemp Oncol (Pozn) 2011; 15: 367-71.

10. Brüning A, Mylonas I. New emerging drugs targeting the genomic integrity and replication machinery in ovarian cancer. Arch Gynecol Obstet. 2011; 283: 1087-96.

11. Mabuchi S, Hisamatsu T, Kawase C, et al. The activity of trabectedin as a single agent or in combination with everolimus for clear cell carcinoma of the ovary. Clin Cancer Res 2011; 17: 4462-73.
12. Martínez-Serra J, Maffiotte E, Martín J, et al. Yondelis ${ }^{\circledR}$ (ET-743, Trabectedin) sensitizes cancer cell lines to CD95-mediated cell death: new molecular insight into the mechanism of action. Eur J Pharmacol 2011; 658: 57-64.

13. Soares DG, Escargueil AE, Poindessous V, Sarasin A, de Gramont A, Bonatto D, Henriques JA, Larsen AK. Replication and homologous recombination repair regulate DNA double-strand break formation by the antitumor alkylator ecteinascidin 743. Proc Natl Acad Sci U S A 2007; 104: 13062-7.

14. Australian Public Assessment Report for Trabectedin, 2010. Available at: http://www.tga.gov.au/pdf/auspar/auspar-yondelis.pdf.

15. Sessa C, De Braud F, Perotti A, et al. Trabectedin for women with ovarian carcinoma after treatment with platinum and taxanes fails. J Clin Oncol 2005; 23: 1867-74.

16. Krasner CN, McMeekin DS, Chan S, et al. A Phase II study of trabectedin single agent in patients with recurrent ovarian cancer previously treated with platinum-based regimens. $\mathrm{Br}$ J Cancer 2007; 97: 1618-24.

17. Thertulien R, Manikhas GM, Dirix LY, et al. Effect of trabectedin on the QT interval in patients with advanced solid tumor malignancies. Cancer Chemother Pharmacol 2012; 69: 341-50.

18. Pardo B, Salazar R, Ciruelos E, et al. Phase I and pharmacokinetic study of trabectedin 3-hour infusion every three weeks in patients with advanced cancer and alteration of hepatic function. Med Oncol 2012; 29: 2240-50.

19. Green AE, Rose PG. Pegylated liposomal doxorubicin in ovarian cancer. Int I Nanomedicine 2006; 1: 229-39.

20. Nomura H, Tsuda H, Kataoka F, Chiyoda T, Yamagami W, Tominaga E, Susumu N, Aoki D. Retrospective study comparing irinotecan and pegylated liposomal doxorubicin in treatment of recurrent platinum-refractory/resistant epithelial ovarian cancer. Eur J Gynaecol Oncol 2012; 33: 86-9.

21. Monk BJ, Herzog TJ, Kaye SB, et al. Final survival results of the randomized phase III study of trabectedin with pegylated liposomal doxorubicin (PLD) versus PLD in recurrent ovarian cancer. J Clin Oncol 2011; 29 suppl. 15: abstract 5046.

22. Monk BJ, Herzog TJ, Kaye SB, et al. Trabectedin plus pegylated liposomal doxorubicin (PLD) versus PLD in recurrent ovarian cancer: overall survival analysis. Eur J Cancer 2012; 48: 2361-8.

23. Krasner CN, Poveda A, Herzog TJ, et al. Patient-reported outcomes in relapsed ovarian cancer: results from a randomized Phase III study of trabectedin with pegylated liposomal doxorubicin (PLD) versus PLD alone. Gynecol Oncol 2012; 127: 161-7.

24. Klasa-Mazurkiewicz D, Jarząb M, Milczek T, Lipińska B, Emerich J. Clinical significance of VEGFR-2 and VEGFR-3 expression in ovarian cancer patients. Pol J Pathol 2011; 62: 31-40.

25. Czerkierdowski A, Czerkierdowska S, Daniłoś J. Vasculogenic mimicry in malignant ovarian tumours. Prz Menopauz 2011; 15: 95-101.

26. Kroep JR. Advances in epithelial ovarian cancer therapy. Curr Pharm Des 2012; 18: 3735-40.

27. Takano M, Ikeda Y, Kudoh K, Kita T, Sasaki N, Kikuchi Y. Complete remission of recurrent ovarian clear cell carcinoma by chemotherapy with bevacizumab, trabectedin and oxaliplatin. J Obstet Gynaecol Res 2013; 39: 872-5.

28. Menchaca R, Martínez V, Rodríguez A, Rodríguez N, Flores M, Gallego P, Manzanares I, Cuevas C. Synthesis of natural ecteinascidins (ET-729, ET-745, ET-759B, ET-736, ET-637, ET-594) from cyanosafracin B. J Org Chem 2003; 68: 8859-66.

\section{Address for correspondence}

\section{Agnieszka Marczak}

\section{Department of Thermobiology}

Faculty of Biology and Environmental Protection

University of Lodz

Pomorska 141/143

90-236 Lodz, Poland

e-mail: aszwar@biol.uni.lodz.pl

Submitted: 20.11.2012

Accepted: 17.12 .2013 